\title{
Caractérisation phénotypique de la race ovine Rembi d'Algérie
}

\author{
Abbas Laoun ${ }^{1,2}$ Sahraoui Harkat ${ }^{3}$ Rédha Benali ${ }^{3}$ \\ Benalia Yabrir ${ }^{1}$ Acème Hakem ${ }^{1}$ Djamila Ranebi ${ }^{4}$ \\ Abderrahmane Maftah ${ }^{5}$ Toufik Madani ${ }^{6}$ \\ Anne Da Silva ${ }^{* *}$ Mohamed Lafri ${ }^{3 *}$
}

Mots-clés

Ovin Rembi, race d'animal d'élevage, anatomie animale, ressource génétique animale, Algérie

Submitted: March 15, 2015;

Accepted: September 21, 2015;

Published: November 20, 2015

\begin{abstract}
Résumé
Le cheptel ovin algérien reste méconnu, malgré son intérêt économique et les qualités très précieuses des races locales qui montrent des adaptations exceptionnelles dans des conditions environnementales extrêmes. La variabilité phénotypique de la Rembi, race algérienne menacée, a été étudiée au moyen d'une analyse discriminante multivariée. L'échantillonnage a pris en considération la diversité des environnements de production présents dans le pays selon les recommandations de la FAO. Au total 722 femelles et 60 mâles ont été phénotypés sur une grande zone incluant le berceau de la race, en utilisant 21 variables quantitatives et 12 variables qualitatives. Les résultats montrent une forte homogénéité phénotypique qui suggère une homogénéité intraraciale au niveau génétique, à confirmer par des analyses moléculaires. En effet, aucune structuration phénotypique n'a été mise en évidence, que ce soit en considérant le statut de la ferme (privée/étatique), la zone climatique (aride/semi-aride/subhumide), la mobilité du troupeau (sédentaire/semi-sédentaire/transhumant) ou encore la variété. Seule la considération du facteur région a permis la mise en évidence de sous-groupes au sein de la race. Les échanges commerciaux, organisés autour de marchés principaux, favorisent les flux d'individus à l'intérieur des différentes régions et semblent responsables d'un modèle phénotypique unique. Ces résultats permettent pour la première fois de caractériser finement la Rembi. Les indices morphologiques la situent notamment parmi les races à viande. Cette étude est la première étape vers la mise en place de programmes de conservation de cette race très menacée par des croisements avec la OuledDjellal, la race algérienne dominante.
\end{abstract}

- Pour citer cet article : Laoun A., Harkat S., Benali R., Yabrir B., Hakem A., Ranebi D., Maftah A., Madani T., Da Silva A., Lafri M., 2015. Phenotypic characterization of the Rembi sheep of Algeria [in French]. Rev. Elev. Med. Vet. Pays Trop., 68 (1): 19-26
1. Université de Djelfa, Algérie.

2. Ecole nationale supérieure vétérinaire d'El-Harrach, Alger, Algérie.

3. Institut des sciences vétérinaires, Université de Blida, Algérie.

4. Institut technique de l'élevage, Birtouta, Algérie.

5. INRA, UMR Génétique moléculaire animale, université de Limoges, 87000 Limoges, France.

6. Université de Setif, El Bez, Algérie.

* Auteur pour la correspondance

Tél. : +33 (0)5 55457675 ; fax : + 33 (0)5 55457653

E-mail : anne.blondeau@unilim.fr

† Les auteurs ont contribué à part égale à la réalisation de l'étude.

\section{INTRODUCTION}

Le cheptel ovin algérien compte 21,4 millions de têtes représentant environ $80 \%$ du stock d'animaux d'élevage (MADR/DSASI, 2010 ; ONS, 2014). Les ovins représentent une valeur économique loin d'être négligeable en Algérie. En effet, le mouton est l'un des rares animaux capable de tirer profit des environnements hostiles (steppes, hauts plateaux, déserts) rencontrés dans le pays. Aussi l'activité ovine occupe-t-elle une position clé dans l'économie nationale (Boutonnet, 2003). Elle constitue la majeure partie du revenu de plus d'un tiers de la population (Chellig, 1992). Chaque année, 7,5 millions de têtes de bétail, issues de la production nationale, sont destinées à la boucherie pour un total de 260000 tonnes équivalent carcasse (MADR, 2007). Pourtant, l'Algérie est loin d'assurer son autosuffisance que ce soit pour les viandes rouges ou le lait. La viabilité du secteur de l'élevage est largement dépendante de l'amélioration des méthodes de production qui tarde à se concrétiser. 
La mise en place de pratiques de conduite plus efficaces ne pourra être effective qu'une fois le cheptel ovin algérien rigoureusement caractérisé d'un point de vue phénotypique et génétique. Sur le plan phénotypique, l'étude de référence de Chellig (1992) apporte des éléments préliminaires descriptifs relatifs aux caractéristiques morphologiques des races ovines algériennes. Sur le plan génétique, la récente étude de Gaouar et al. (2015) donne une image de la diversité génétique du cheptel ovin algérien mais n'apporte pas d'élément sur la structuration fine d'une race en particulier. La prédominance d'une race sur le marché est l'une des menaces principales pesant sur la diversité génétique de l'élevage ovin algérien. En effet, parmi les huit races majeures, Rembi, OuledDjellal, Hamra, Sidaoun, D'men, Berbère, Barbarine, Taadmit, la préférence des éleveurs pour la Ouled-Djellal peut être considérée comme une pression forte qui tend à homogénéiser le cheptel ovin algérien. La situation est ainsi devenue critique pour les autres races qui sont délaissées et/ou soumises à des pratiques de croisements avec la race dominante.

La Rembi est tout particulièrement menacée par ces pratiques (Matet, 2009) ; la dilution génétique causée par ces croisements est telle que la race a perdu, pour une large part, son originalité génétique (Gaouar et al., 2015). D’un point de vue phénotypique, les spécimens tels qu'ils étaient décrits par Chellig (1992), à savoir caractérisés par une robe de couleur fauve, sont désormais très rares et remplacés par des individus présentant une robe blanche (figures 1 et 2), proche de celle de la Ouled-Djellal. La Rembi est

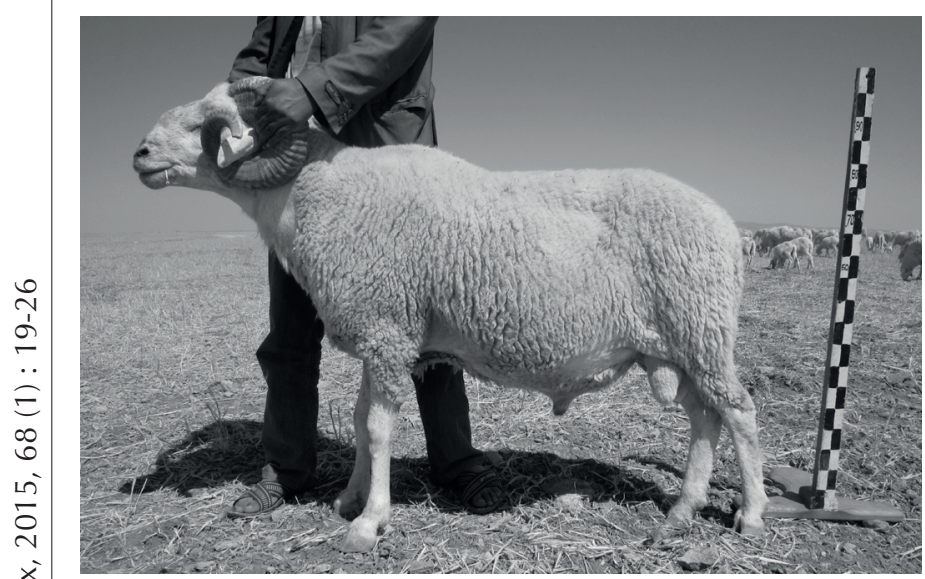

Figure 1 : bélier Rembi photographié à Djelfa en Algérie en 2014 (C) A. Laoun).

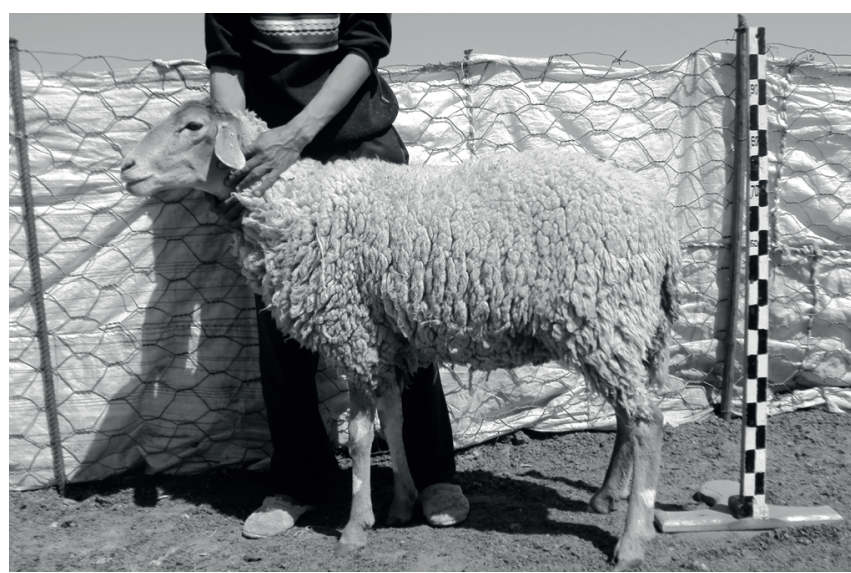

Figure 2 : brebis Rembi photographiée à Tiaret en Algérie en 2014 (@) A. Laoun). issue probablement de croisements entre la Ouled-Djellal et le mouton sauvage du Djebel Amour (Chellig, 1992). La race compte deux millions de têtes (FAO DAD-IS 2003, www.fao.org/dad-is). Sa localisation géographique est largement centrée dans la région de Tiaret et les régions avoisinantes (ce qui correspond au berceau de la race), limitée à l'ouest par le Chott Chergui, à l'est par l'Oued Touil, au nord par Tiaret, et au sud par Aflou et El Bayadh (Chellig, 1992). Cette race rustique est réputée pour être particulièrement bien adaptée aux conditions de vie difficiles des hauts plateaux steppiques.

La caractérisation des races est essentielle pour une gestion optimale et durable des ressources génétiques animales (Lanari et al., 2003). La première étape de cette caractérisation implique la définition des races par une description morphologique rigoureuse (Gizaw et al. 2007). L'objectif de l'étude a été d'obtenir une caractérisation morphologique fine de la Rembi, au travers d'une analyse multivariée discriminante, comme première étape dans la conservation et l'amélioration génétique de la race. Selon les recommandations de la FAO (2011), la description de la race a été réalisée en considérant l'environnement naturel et de production des cheptels, ce qui est particulièrement important dans les pays présentant une grande variété de systèmes de production.

\section{MATERIEL ET METHODES}

\section{Echantillonnage des animaux}

L'étude a été réalisée avec 722 femelles et 60 mâles de race Rembi, échantillonnés parmi les trois régions administratives algériennes de Djelfa, Laghouat et Tiaret (figure 3), correspondant au berceau de la race et aux zones dans lesquelles se trouve la majorité des ovins Rembi (MADR/DSASI, 2010 ; ONS, 2014). L'échantillonnage a été réalisé dans un rayon de 130 kilomètres autour de la ville de Ksar Chellala. Au total, 23 fermes ont été visitées (le tableau supplémentaire [suppl.] SI montre plus de détails sur l'échantillonnage). L'échantillonnage a concerné des élevages uniquement

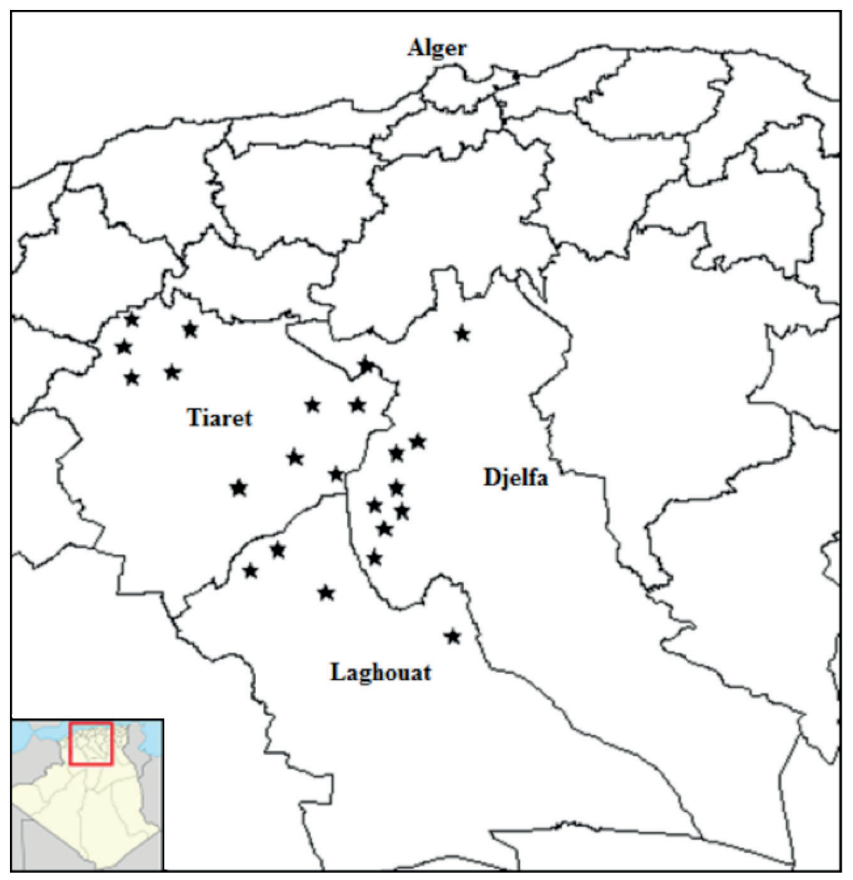

Figure 3 : localisation des cheptels échantillonnés en Algérie; l'échantillonnage inclut le berceau de la race ovine Rembi. 
composés d'ovins de race Rembi. D'après les éleveurs, cinq variétés de cette race peuvent être définies sur la base de critères morphologiques : la Rembi (cette variété porte donc le même nom que la race), la Sagaa, la Djelfabotma, la Karnacha et la Chagra.

Les données ont été collectées durant le printemps 2013. Dans chaque région, des éleveurs privés ont été sélectionnés aléatoirement. Dans chaque ferme, un groupe de femelles non gravides (les ostrus n'étaient pas synchronisés) ont été sélectionnées, puis les femelles à phénotyper ont été désignées de manière aléatoire. Tous les individus phénotypés étaient âgés de 36 mois au moins, l'âge étant estimé en fonction de la dentition (Wilson et Durkin, 1984). Les variables qualitatives et quantitatives (respectivement 21 et 12) utilisées pour le phénotypage ont été répertoriées respectivement dans les tableaux suppl. SII et SIII.

La plupart des traits étudiés ont été adaptés à partir de la liste des descripteurs ovins, publiés par la FAO (2011). Les brebis ont été pesées avec un peson électronique portable, à 0,01 kilogramme près. Toutes les mesures ont été réalisées par le même groupe d'opérateurs, de façon à éviter les biais inhérents à l'introduction d'une variabilité interindividuelle. Pour obtenir les mensurations, les opérateurs, qui ont disposé les animaux sur un sol horizontal, ont utilisé un ruban métrique, une toise et un pied à coulisse. Chaque éleveur a rempli un questionnaire établi selon les recommandations de la FAO (2011), pour recueillir des informations concernant l'environnement de production des élevages.

\section{Environments de production}

\section{Conduite des cheptels}

Deux types de fermes ont été visités. D'une part, les fermes appartenant à l'Etat qui peuvent être organisées en fermes pilotes (habilitées à faire le commerce de leurs spécimens) ou celles de l'Institut technique de l'élevage dont l'objet est l'étude et la préservation de races, et, d'autre part, les fermes privées.

Le système d'élevage algérien demeure largement traditionnel. Si l'on considère la mobilité des cheptels, trois types de conduites sont distingués : (a) les troupeaux sédentaires, (b) les troupeaux semi-sédentaires, utilisant une ou deux pâtures saisonnières localisées à moins de 50 kilomètres de l'aire principale d'élevage, et (c) les troupeaux transhumants qui pâturent en hiver généralement dans les régions sahariennes et qui sont menés en été sur les lieux les plus propices en fonction de la disponibilité en eau du moment (régions céréalières de Tiaret et Sétif).

\section{Environnement naturel}

Il existe en Algérie cinq zones bioclimatiques majeures allant de la plus aride à la plus humide, basées essentiellement sur les précipitations et les températures (hivernales en particulier). Notre échantillonnage était localisé principalement au niveau de l'Atlas saharien, caractérisé par des altitudes décroissantes d'ouest en est, et sur les hauts plateaux. L'aire d'échantillonnage a été découpée selon trois zones climatiques définies par Le Houérou et al. (1977), et Le Houérou (2004) : (a) la zone subhumide, située à 600-900 m d'altitude, au nord de l'Atlas tellien, caractérisée par des forêts de chênes verts (Quercus ilex) et de pins d'Alep (Pinus halepensis) (Meddour, 2010), (b) la zone semi-aride avec des précipitations annuelles comprises entre 200 et $500 \mathrm{~mm}$, sur les pentes sud de l'Atlas tellien, caractérisée par des forêts plus ou moins dégradées et des maquis à Quercus rotundifolia et Callitris articulata qui font partie des essences communément rencontrées avec les pins d'Alep en altitude, et (c) la zone aride, avec une pluviométrie annuelle comprise entre 0 et $200 \mathrm{~mm}$, dont les steppes, plus au sud voire présahariennes présentent un couvert végétal pauvre sur sol souvent dégradé. Dans cette zone les espèces forestières ont disparu et les espèces végétales sont caractéristiques de la steppe, comme l'armoise (Artemisia vulgaris), l'alfa (Stipa tenacissima) et le sparte (Lygeum spartum).

\section{Indices morphologiques}

A partir des mesures unitaires (tableau suppl. SII), des indices ont été calculés en suivant les méthodes de Salako (ONS, 2014) et d'Alderson (1999) visant à déterminer le type et la fonction de la race. Ces indices ont été calculés comme suit :

Indice poids (sans unité $)=[($ longueur totale du corps $\mathrm{x}$ tour de poitrine) $\times$ (largeur des hanches + largeur de la poitrine) $] / 1050$

Indice pente $(\mathrm{cm})$ = hauteur au garrot - hauteur au sacrum

Indice longueur (sans unité) = longueur totale du corps / hauteur au garrot

Indice largeur (sans unité) = largeur des hanches / largeur de la poitrine

Indice profondeur (sans unité) = profondeur de la poitrine / hauteur au garrot

Indice hauteur $(\mathrm{cm})=$ hauteur au garrot - profondeur de la poitrine

Balance $($ sans unité $)=($ largeur du bassin $\times$ largeur des hanches $) /$ (profondeur de la poitrine $\times$ largeur de la poitrine)

Indice cumulé $($ sans unité $)=($ indice poids / moyenne du poids $)+$ (indice longueur + balance)

\section{Analyses statistiques}

Mâles et femelles ont été analysés séparément du fait du dimorphisme et des tailles d'échantillons différentes. Les femelles ont représenté l'échantillon le plus important, ce qui a permis d'affiner l'analyse statistique. L'objectif principal a été de déterminer dans quelle mesure les caractères morphologiques de la race présentaient ou non des variations significatives en fonction (a) de la région échantillonnée, (b) de la zone climatique, (c) de la variété, et (d) des pratiques de conduite.

Pour les variables quantitatives, les moyennes, erreurs types et coefficients de variation ont été estimés pour tous les traits (tableau I). Une analyse discriminante linéaire (ADL) a été menée afin de déterminer quelles variables morphométriques étaient les plus à même de discriminer les régions, les zones climatiques, les variétés ou encore les pratiques de conduite. L'importance relative de ces variables a été étudiée au seuil de 0,001.

La capacité des fonctions canoniques à assigner, avec justesse, chaque individu à son groupe d'appartenance a été évaluée par le calcul des pourcentages d'assignements corrects, en l'utilisant une procédure de type analyse discriminante. Des analyses de variance complétées par des contrastes de Dunnett ont permis de comparer les valeurs moyennes quantitatives.

Pour les variables qualitatives, les fréquences ont été calculées pour les différentes modalités de chaque caractère discret analysé. La capacité des fonctions canoniques à assigner, avec justesse, chaque individu à son groupe d'appartenance a été évaluée par le calcul des pourcentages d'assignements corrects. Les logiciels $\mathrm{R}$ version 3.0.1 (R Core Team, 2012) et Tanagra version 1.4.50 (Rakotomalala, 2005) ont permis de mener l'ensemble des analyses.

\section{RESULTATS}

Parmi les 23 fermes visitées, 6 appartenaient à l'Etat et 17 étaient privées, 9 étaient situées dans la région de Djelfa, 10 dans celle de 


\section{Tableau I}

Moyennes (Moy.) et erreurs types (ET) pour les mesures morphométriques obtenues chez les ovins Rembi d'Algérie et coefficients de variation (CV)

\begin{tabular}{|c|c|c|c|c|}
\hline \multirow[t]{2}{*}{ Mensurations } & \multicolumn{2}{|c|}{ Femelles } & \multicolumn{2}{|c|}{ Mâles } \\
\hline & Moyenne $\pm \mathrm{ET}$ & CV & Moyenne $\pm \mathrm{ET}$ & CV \\
\hline Poids (kg) & $53,22 \pm 0,36^{a}$ & 0,1813 & $84,27 \pm 1,79 b$ & 0,1644 \\
\hline Longueur de la tête (cm) & $23,06 \pm 0,05^{a}$ & 0,0604 & $27,07 \pm 0,21^{b}$ & 0,0608 \\
\hline Largeur des oreilles $(\mathrm{cm})$ & $8,30 \pm 0,03^{a}$ & 0,0867 & $8,54 \pm 0,10^{a}$ & 0,0898 \\
\hline Longueur du cou $(\mathrm{cm})$ & $30,07 \pm 0,14^{\mathrm{a}}$ & 0,1225 & $32,55 \pm 0,50^{b}$ & 0,1191 \\
\hline Longueur du corps $(\mathrm{cm})$ & $107,61 \pm 0,25^{a}$ & 0,0634 & $121,32 \pm 1,14^{b}$ & 0,0730 \\
\hline Tour de la poitrine $(\mathrm{cm})$ & $91,89 \pm 0,26^{a}$ & 0,0766 & $107,08 \pm 0,74^{b}$ & 0,0533 \\
\hline Largeur externe du poitrail (cm) & $18,29 \pm 0,07^{a}$ & 0,1018 & $22,33 \pm 0,23 b$ & 0,0805 \\
\hline Longueur du bassin $(\mathrm{cm})$ & $22,62 \pm 0,08^{a}$ & 0,0896 & $26,07 \pm 0,29 b$ & 0,0865 \\
\hline Largeur des ischions (cm) & $16,42 \pm 0,05^{a}$ & 0,0889 & $18,24 \pm 0,23 b$ & 0,0963 \\
\hline Hauteur au garrot $(\mathrm{cm})$ & $77,32 \pm 0,12^{a}$ & 0,0421 & $88,62 \pm 0,61^{b}$ & 0,0531 \\
\hline Largeur de la tête $(\mathrm{cm})$ & $9,38 \pm 0,02{ }^{a}$ & 0,0599 & $11,49 \pm 0,10^{b}$ & 0,0683 \\
\hline Longueur des oreilles (cm) & $15,62 \pm 0,06^{a}$ & 0,1008 & $15,36 \pm 0,21^{a}$ & 0,1058 \\
\hline Longueur des cornes * (cm) & - & - & $61,75 \pm 3,65$ & 0,4576 \\
\hline Profondeur de la poitrine $(\mathrm{cm})$ & $34,14 \pm 0,07^{a}$ & 0,0522 & $40,94 \pm 0,27^{b}$ & 0,0518 \\
\hline Largeur de la poitrine $(\mathrm{cm})$ & $17,83 \pm 0,08^{a}$ & 0,1150 & $21,23 \pm 0,29 b$ & 0,1050 \\
\hline Largeur interne du poitrail (cm) & $11,63 \pm 0,05^{a}$ & 0,1210 & $13,59 \pm 0,23 b$ & 0,1326 \\
\hline Largeur des hanches $(\mathrm{cm})$ & $18,80 \pm 0,05^{a}$ & 0,0691 & $21,42 \pm 0,22^{b}$ & 0,0810 \\
\hline Tour du canon $(\mathrm{cm})$ & $7,93 \pm 0,05^{a}$ & 0,1586 & $11,05 \pm 1,59 b$ & 1,1131 \\
\hline Longueur de la queue (cm) & $38,71 \pm 0,24^{\mathrm{a}}$ & 0,1641 & $44,49 \pm 0,91^{b}$ & 0,1585 \\
\hline Tour scrotal $*(\mathrm{~cm})$ & - & - & $33,20 \pm 0,38$ & 0,0878 \\
\hline Profondeur scrotale * $(\mathrm{cm})$ & - & - & $21,44 \pm 0,44$ & 0,1576 \\
\hline
\end{tabular}

${ }^{a}, b$ Les moyennes sur une même ligne suivies de lettres différentes sont significativement différentes $(p<0,05)$.

* Paramètres considérés seulement pour les mâles.

Tiaret et 4 dans celle de Laghouat, enfin, 13 élevages étaient de type sédentaire, 7 de type semi-sédentaire et 3 de type transhumant. Ces fermes étaient réparties dans les trois zones bioclimatiques : 6 en zone subhumide, 11 en zone semi-aride et 6 en zone aride. Par ailleurs, 14 fermes étaient spécialisées dans l'élevage de la variété Rembi, 6 de la variété Sagaa, 1 de la variété Djelfabotma, 1 de la variété Karnacha et 10 de la variété Chagra.

\section{Analyse des traits quantitatifs chez les femelles}

En considérant le statut de la ferme (privé/étatique) l'ADL a révélé une valeur du lambda de Wilks de 0,65 ; en considérant le mode d'élevage (sédentaire/semi-sédentaire/transhumant) cette valeur a été proche de 0,56 ; en considérant la zone climatique (aride/semi-aride/ subhumide) elle a été de 0,44 ; et en considérant la variété elle a été de 0,9, sachant que seules les Rembi et les Sagaa ont été prises en compte dans le traitement du facteur variété. En effet les autres variétés n'étant représentées que par un seul élevage, l'échantillonnage ne permettait pas de distinguer entre l'effet variété et l'effet troupeau.

L'ADL a montré pour le facteur région une valeur modérée proche de 0,22 pour le lambda de Wilks, indiquant que la combinaison des variables morphobiométriques pouvait expliquer $78 \%$ de la variance. Des analyses plus poussées relatives à la structuration entre les trois régions échantillonnées ont été menées. L'ADL a été conduite avec la méthode du forward stepping. Les résultats ont montré que sur 19 mesures, 12 étaient significatives $(\mathrm{p}<0,001)$. Les dix variables montrant le meilleur pouvoir de discrimination (selon la valeur de F) sont détaillées dans le tableau II. Les contrastes de Dunnett ont permis de montrer a) dans la région de Tiaret des femelles significativement plus légères, caractérisées par les plus fortes valeurs moyennes pour la largeur des oreilles, la longueur de la tête et la longueur du cou, b) dans la région de Djelfa des femelles aux corps plus longs et des hauteurs au garrot plus importantes et c) dans la région de Laghouat des femelles présentant des valeurs moyennes plus importantes pour la largeur externe du poitrail, la largeur du bassin, le tour de poitrine et la largeur des ischions.

L'ADL a été conduite en considérant ces 12 variables. Les résultats ont montré clairement que les trois régions correspondaient à des groupes distincts (Wilks' Lambda $=0,28$; Bartlett $\chi^{2}=899,20$; $\mathrm{p}=0,000 ;$ Rao $\mathrm{F}=51,80 ; \mathrm{p}=0,000)$. Deux fonctions discriminantes ont permis de retenir $100 \%$ de la variabilité : fonction 1 , eigenvalue $=1,18$ avec $\operatorname{Rc}^{2}=0,74 ;$ fonction 2 : eigenvalue $=0,61$ avec $\mathrm{Rc}^{2}=0,62$. La première fonction retient à elle seule $66 \%$ de la variabilité. La figure 4 permet de visualiser les résultats de l'ADL réalisée en considérant le facteur région. Le pourcentage d'individus correctement assignés dans chaque région est recensé dans le tableau III. En moyenne $83 \%$ des ovins ont été correctement assignés. Inversement, pour chaque région les pourcentages d'assignations incorrectes ont été proches de $20 \%$.

\section{Analyse des caractères discrets chez les femelles}

Quel que soit le facteur considéré les variables discrètes n'apparaissent que faiblement liées à celui-ci (test V de Cramer $<0,15$ ). La capacité des fonctions canoniques à assigner correctement chaque individu dans son groupe d'origine a été estimée en calculant 


\section{Tableau II}

Moyennes (Moy.) et erreurs types (ET) pour les mesures morphométriques des brebis Rembi d'Algérie selon les régions

\begin{tabular}{|c|c|c|c|}
\hline Mensurations & $\begin{array}{c}\text { Tiaret } \\
\text { Moy. } \pm \text { ET } \\
(n=320)\end{array}$ & $\begin{array}{c}\text { Djelfa } \\
\text { Moy. } \pm \text { ET } \\
(n=273)\end{array}$ & $\begin{array}{c}\text { Laghouat } \\
\text { Moy. } \pm \text { ET } \\
(n=129)\end{array}$ \\
\hline Poids (kg) & $48,82 \pm 0,58^{a}$ & $57 \pm 0,58^{b}$ & $56,13 \pm 0,67 b$ \\
\hline Longueur de la tête $(\mathrm{cm})$ & $23,59 \pm 0,07^{a}$ & $22,87 \pm 0,07^{b}$ & $22,14 \pm 0,15^{b}$ \\
\hline Largeur des oreilles $(\mathrm{cm})$ & $8,61 \pm 0,03$ a & $8,13 \pm 0,05 b$ & $7,91 \pm 0,05 b$ \\
\hline Longueur du cou $(\mathrm{cm})$ & $31,62 \pm 0,16^{a}$ & $28,77 \pm 0,14^{b}$ & $28,98 \pm 0,51^{b}$ \\
\hline Longueur totale du corps $(\mathrm{cm})$ & $107,58 \pm 0,36^{b}$ & $109,67 \pm 0,38^{a}$ & $103,35 \pm 0,60^{b}$ \\
\hline Tour de poitrine $(\mathrm{cm})$ & $89,9 \pm 0,28^{b}$ & $93,29 \pm 0,34^{b}$ & $95,11 \pm 0,38^{a}$ \\
\hline Largeur externe du poitrail $(\mathrm{cm})$ & $18,3 \pm 0,11^{b}$ & $17,71 \pm 0,10^{b}$ & $19,49 \pm 0,13^{a}$ \\
\hline Largeur du bassin $(\mathrm{cm})$ & $22,32 \pm 0,11^{b}$ & $22,23 \pm 0,10^{b}$ & $24,16 \pm 0,19^{a}$ \\
\hline Largeur des ischions $(\mathrm{cm})$ & $16,09 \pm 0,08^{b}$ & $16,39 \pm 0,06^{b}$ & $17,33 \pm 0,17^{a}$ \\
\hline Hauteur au garrot $(\mathrm{cm})$ & $76,9 \pm 0,18^{b}$ & $78,46 \pm 0,18^{a}$ & $75,94 \pm 0,29 b$ \\
\hline
\end{tabular}

${ }^{a, b}$ Les moyennes sur une même ligne suivies de lettres différentes sont significativement différentes $(p<0,05)$.

le pourcentage d'assignement correct dans chaque groupe. Les matrices de confusion ont montré des taux d'erreur très élevés quel que soit le facteur (tableau IV). Ces analyses ont clairement postulé en faveur d'une forte homogénéité entre les individus Rembi, considérant les variables discrètes (tableau III).

\section{Indices morphologiques}

Les fréquences relatives aux variables qualitatives sont recensées pour les mâles et pour les femelles dans le tableau suppl. SIV. Les valeurs moyennes concernant chaque variable quantitative et les

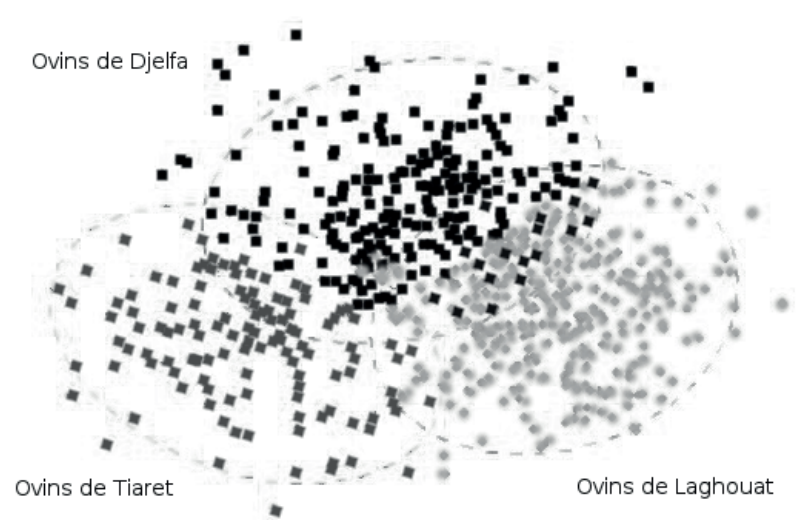

Figure 4 : analyse discriminante linéaire réalisée en fonction des trois régions échantillonnées en Algérie (Tiaret, Djelfa et Laghouat) et ellipses (95\% de confiance), pour la race Rembi.

\section{Tableau III}

Brebis Rembi d'Algérie (\%) classées en fonction des variables quantitatives au sein des trois régions échantillonnées

\begin{tabular}{lccc} 
& $\begin{array}{c}\text { Tiaret } \\
(\mathbf{n = 3 2 0})\end{array}$ & $\begin{array}{c}\text { Djelfa } \\
(\mathbf{n = 2 7 3})\end{array}$ & $\begin{array}{c}\text { Laghouat } \\
(\mathbf{n = 1 2 9})\end{array}$ \\
\hline Djelfa & 78,75 & 14,29 & 6,96 \\
Tiaret & 12,19 & 85,62 & 2,19 \\
Laghouat & 9,30 & 10,08 & 80,62
\end{tabular}

coefficients de variation (CV) sont reportés dans le tableau I pour les mâles et les femelles. Enfin, les indices morphologiques sont consignés pour les mâles et les femelles dans le tableau $\mathrm{V}$.

\section{Tableau IV}

En considérant les différents facteurs, pourcentage d'erreur pour chaque matrice de confusion dans I'analyse des traits qualitatifs des brebis Rembi d'Algérie

\begin{tabular}{lc} 
Facteur & \% d'erreur \\
\hline Variété $^{1}$ & 68,8 \\
Région $^{2}$ & 52,4 \\
Statut $^{3}$ & 45,4 \\
Zone climatique $^{4}$ & 38,1
\end{tabular}

1. Rembi/Sagaa ; 2. Djelfa/Tiaret/Laghouat ; 3. privé/étatique ; 4. subhumide/aride/ semi-aride

\section{Tableau V}

Moyennes (Moy.) et erreurs types (ET) pour les indices de développement corporel des ovins Rembi d'Algérie

\begin{tabular}{lcc} 
& \multicolumn{1}{c}{ Femelles } & Mâles \\
\cline { 2 - 3 } & Moy. \pm ET & Moy. \pm ET \\
\hline Poids/1050 & $173,28 \pm 27,34^{a}$ & $264,99 \pm 39,30^{b}$ \\
Indice du dos & $1,27 \pm 1,46^{a}$ & $2,24 \pm 1,80^{\mathrm{b}}$ \\
Indice de longueur & $1,39 \pm 0,08^{a}$ & $1,37 \pm 0,09^{a}$ \\
Indice de largeur & $1,07 \pm 0,11^{a}$ & $1,02 \pm 0,11^{b}$ \\
Indice de profondeur & $0,44 \pm 0,02^{a}$ & $0,46 \pm 0,02^{b}$ \\
Indice de hauteur & $43,18 \pm 2,89^{a}$ & $47,69 \pm 3,78^{b}$ \\
Balance & $0,70 \pm 0,08^{a}$ & $0,65 \pm 0,08^{b}$ \\
Indice cumulé & $5,35 \pm 0,5340^{a}$ & $5,16 \pm 0,5163^{b}$
\end{tabular}

a, $\mathrm{b}$ Les moyennes sur une même ligne suivies de lettres différentes sont significativement différentes $(\mathrm{p}<0,05)$. 


\section{DISCUSSION}

La race Rembi a été caractérisée d'un point de vue morphologique en considérant 21 variables quantitatives et 12 variables qualitatives. L'échantillonnage conséquent de femelles Rembi dans une vaste zone comprenant le berceau de la race a permis d'étudier finement, au travers d'analyses canoniques discriminantes, la structuration de la race en Algérie. La considération des variables qualitatives n'a montré aucune structuration phénotypique quel que soit le facteur considéré (statut de la ferme, zone climatique, mobilité du troupeau, région ou variété). Ces analyses ont postulé en faveur d'une forte homogénéité intrarace, considérant les traits discrets.

De même, les variables quantitatives n'ont pas montré de structuration forte, excepté pour le facteur région pour lequel les brebis ont présenté des distinctions nettes. Il est à noter que l'analyse sur les deux variétés, Rembi et Sagaa, permet de conclure que le concept de variété n'était pas appuyé par une réalité phénotypique pour ce qui concerne la race Rembi. Les entretiens avec les éleveurs ont révélé que le terme de variété était souvent employé pour désigner un troupeau transmis de génération en génération. Parfois une variété peut montrer des particularités phénotypiques marquées : la Sagaa par exemple est caractérisée par des tâches sombres au niveau du cou.

Ces résultats suggèrent que le seul facteur laissant apparaître une structuration phénotypique était la région. En effet, au sein de chaque région, un ou deux marchés majeurs (Djelfa ville et Hassi Bahbah pour la région de Djelfa, Sougueur pour la région de Tiaret, et Laghouat-ville pour la région de Laghouat) sont principalement fréquentés par les éleveurs de la région en question. Les flux de gènes sont ainsi largement circonscrits à l'intérieur de chaque région.

La variabilité morphologique peut être considérée comme un bon indicateur de la variabilité génétique et ainsi du potentiel adaptatif de la race (Toro et al., 2011). La variabilité génétique intraraciale est cruciale, seule à même de permettre à la race de s'adapter à des environnements naturels et de production changeants (Meuwissen, 2009), et de répondre de manière significative à la sélection artificielle (Toro et Caballero, 2005). Nos résultats ne sont pas particulièrement optimistes en termes de diversité génétique. En effet, en dépit de la diversité marquée des environnements naturels et de production algériens, et bien que les cheptels n'aient jamais été soumis à une sélection artificielle intense (la conduite des cheptels reste fortement traditionnelle), aucune structuration phénotypique n'a pu être mise en évidence, excepté lorsque le découpage en régions de la zone échantillonnée a été considéré. Ainsi, le modèle phénotypique détecté apparaît uniquement dépendant des modes d'échanges économiques.

Même si la caractérisation morphologique d'une race permet d'avoir des éléments de réponse concernant la diversité génétique de ladite race (Al-Rawi et Al-Athar, 2000 ; Nsoso et al., 2004), ces résultats doivent être complétés par des analyses moléculaires réalisées également sur un échantillonnage fin d'individus, qui prend en compte les différents environnements naturels et de production (FAO, 2011).

La définition morphologique de la race est nécessaire à la connaissance de celle-ci (Rothschild, 2003). D'après les variables discrètes considérées, les mâles et les femelles Rembi peuvent être décrits comme suit (figures 1 et 2) : les oreilles sont généralement longues (sous la commissure labiale), la queue arrive au niveau du jarret, le dos est subconcave, la tête et les membres sont mouchetés de tâches fauves alors que les flancs sont unis d'une couleur blanche tirant sur le jaune. Le profil du chanfrein est droit ou légèrement incurvé chez les femelles et incurvé chez les mâles. La toison est semi-invasive chez les femelles et généralement invasive chez les mâles. Les trayons sont semi-horizontaux.

Les mesures quantitatives permettent d'obtenir une caractérisation morphologique plus précise. Le dimorphisme est marqué avec des mâles qui présentent des traits de dimensions supérieures en moyenne aux femelles. La race est caractérisée par une hauteur au garrot supérieure à la hauteur prise au niveau de la croupe. Considérant les indices calculés par Alderson (1999) et Salako (2006), l'indice du dos qui correspond à l'inclinaison prend une valeur modérée chez les femelles $(1,2 \mathrm{~cm})$ et plus marquée chez les mâles $(2,2 \mathrm{~cm})$, l'indice de longueur, proche de 1,3 chez les mâles et les femelles, caractérise une race présentant un corps allongé, l'indice de largeur, proche de 1 chez les mâles et les femelles, montre que les largeurs au niveau des hanches et de la poitrine sont très proches, l'indice de profondeur, proche de 0,4 chez les mâles et les femelles, indique une profondeur de poitrine modérée, l'indice de hauteur montre que les animaux sont hauts sur pattes (particulièrement les mâles), et la balance montre une surface légèrement supérieure au niveau de la poitrine par rapport à la croupe. La prise en considération de tous ces indices et de l'indice cumulé indique pour la Rembi une forme globalement rectangulaire, caractéristique des races à viande (Cerqueira et al., 2011).

Si l'on compare les mensurations de la Rembi à celles de races nigérianes (Yakubu et Ibrahim, 2011), d'Afrique du Sud (Mavule, 2013), du Ghana (Birteeb et al., 2013), du Burkina Faso (Traoré et al., 2008) ou de la Zambie (Pareacute et Casanova, 2013), la Rembi se positionne parmi celles au format le plus important. Si l'on compare la Rembi à la Ouled-Djellal, étudiée en Algérie en considérant les mêmes variables par Harkat et al. (2015), on constate, malgré la forte ressemblance des deux races, que la Ouled-Djellal est plus lourde avec un poids moyen de dix kilogrammes environ supérieur, et plus élancée, avec un cou plus long de trois centimètres en moyenne est une hauteur au garrot plus élevée de trois centimètres en moyenne.

\section{- CONCLUSION}

La race Rembi, race réputée rustique, montre une résistance accrue au froid et à la sécheresse, caractéristique d'une adaptation aux conditions de vie des hauts plateaux (Chellig, 1992), est dans une situation de vulnérabilité. En effet les éleveurs désireux d'améliorer la conformation de leurs animaux réalisent des croisements avec la race Ouled-Djellal qui présente des mensurations plus avantageuses. La Rembi est tout particulièrement sujette à ces pratiques qui conduisent à la perte de l'originalité génétique de cette race (Gaouar et al., 2015) (toutefois, les effets de ces croisements n'étaient pas l'objet de cette étude).

La Rembi est très bien adaptée aux conditions montagneuses, contrairement à la Ouled-Djellal qui tend cependant à la supplanter (Harkat et al., 2015), y compris dans des zones qui ne lui conviennent pas, ce qui est rendu possible par l'artificialisation du pastoralisme (complémentation alimentaire) (Kanoun et al., 2007). Cette étude est une première étape qui sera complétée par une étude de la diversité génétique de la race à une échelle fine, afin de mettre en place des plans de conservation pour cette race présentant des traits spécifiques de grand intérêt, à préserver et à valoriser au travers de stratégies de conduite modernes et réfléchies.

\section{Remerciements}

Les auteurs remercient les éleveurs pour leur implication au cours de la campagne d'échantillonnage. L'aide des personnels 
techniques de l'Institut technique de l'élevage de Ksar-Chellala a été particulièrement précieuse et appréciée. Enfin les auteurs remercient le support logistique apporté par les universités de Djelfa et de Blida.

\section{REFERENCES}

Alderson G.L.H., 1999. The development of a system of linear measurements to provide an assessment of type and function of beef cattle. Anim. Genet. Resour. Inf., 25: 45-56

Al-Rawi A.A., Al-Athar A.K., 2000. Characteristics of indigenous chicken in Iraq. Anim. Genet. Res. Inf., 32: 87-94

Birteeb P.T., Peters S.O., Yakubu A., Adeleke M.A., Ozoj M.O., 2013. Multivariate characterisation of the phenotypic traits of Djallonke and Sahel sheep in Northern Ghana. Trop. Anim. Health Prod., 45: 267-274

Boutonnet J.P., 2003. Intensification de la production des petits ruminants : pièges et promesses. FAO, Rome, Italie. http://www.fao.org/wairdocs/ilri/x5520b/x5520b05.htm

Cerqueira J.O.L., Feás X., Iglesia A., Pacheco L.F., Araújo J.P.P., 2011. Morphological traits in Portuguese Bordaleira de Entre Douro e Minho sheep: divergence of the breed. Anim. Prod. Sci., 51 (7): 635-641

Chellig R., 1992. Les races ovines algériennes. Office des publications universitaires, Alger, Algérie

FAO, 2011. Phenotypic characterization of animal genetic resources. FAO, Rome, Italy. (Animal production and health guidelines No 11)

Gaouar S.B.S., Da Silva A., Ciani E., Kdidi S., Aouissat M., Dhimi L., Lafri M., Maftah A., Mehtar N., 2015. Admixture and local breed marginalization threaten Algerian sheep diversity. Plos One, 10 (4), doi: 10.1371/journal.pone.0122667

Gizaw S., Van Arendonk J.A.M., Komen H., Windig J.J., Hanotte O., 2007. Population structure, genetic variation and morphological diversity in indigenous sheep of Ethiopia. Anim. Genet., 38: 621-628

Harkat S., Laoun A., Benali R., Outayeb D., Ferrouk M., Maftah A., Da Silva A., Lafri M., 2015. Phenotypic characterization of the major sheep breed in Algeria. Rev. Med. Vet., (166): 138-147

Kanoun A., Yakhlef H., Cherfaoui M.A., 2007. Système d'élevage et stratégie d'adaptation des élevages ovins en Algérie. Rencontre Rech. Rumin., 14 : 181-184

Lanari M.R., Taddeo H., Domingo E., Centeno M.P., Gallo L., 2003. Phenotypic differentiation of exterior traits in local Criollo goat population in Patagonia Argentina. Arch. Anim. Breed, 46: 347-356

Le Houérou H.N., 2004. An agro-bioclimatic classification of arid lands in the isoclimatic Mediterranean zones. Arid Land Res. Manage., 18: 301-346

Le Houérou H.N., Claudin J., Pouget M., 1977. Etude bioclimatique des steppes algériennes. Bull. Soc. Nat. Afr. Nord, 86: 3-4

MADR, 2007. Statistiques agricoles, superficies et production, Séries A et B. Ministère de I'Agriculture et du Développement rural, Alger, Algérie
MADR/DSASI, 2010. Statistiques élevages, cheptels E2009. Ministère de I'Agriculture et du Développement rural / Direction des statistiques agricoles et des systèmes d'information, Alger, Algérie

MATET, 2009. Quatrième rapport national sur la mise en œuvre de la convention sur la diversité biologique au niveau national. Ministère de I'Aménagement du Territoire, de l'Environnement et du Tourisme, Alger, Algérie

Mavule B.S., 2013. Characterization of Zulu sheep production system: Implications for conservation and improvement. Sci. Res. Essays, 8: 1226-1238

Meddour R., 2010. Bioclimatologie, phytogéographie et phytosociologie en Algérie, exemple des groupements forestiers et pré-forestiers de la Kabylie Djurdjuréenne. Thèse Doct., Université de Tizi Ouzou, Algérie

Meuwissen T.H.E., 2009. Towards a consensus on how to measure neutral genetic diversity? J. Anim. Breed. Genet., 126: 333-334

Nsoso S.J., Podisi B., Otsogile E., Mokhutshwane B.S., Ahmadu B., 2004. Phenotypic characterization of indigenous Tswana goats and sheep breeds in Botswana. Trop. Anim. Health Prod., 36: 789-800

ONS, 2014. L'Algérie en quelques chiffres, résultats 2013. Office national des statistiques, Alger, Algerie

Pareacute S., Casanova P.-C., 2013. Biometrical multivariate study of the Zambian indigenous Fat-tailed sheep. Int. J. Livest. Prod., 4: 148-154

R Core Team, 2012. R: A language and environment for statistical computing. R Foundation for statistical computing, Vienna, Austria. www.R-project.org/

Rakotomalala R., 2005. Tanagra : un logiciel gratuit pour l'enseignement et la recherche. In : Actes $5^{\text {es }}$ journées Extraction et gestion des connaissances, Paris, France, 18-21 janv. 2005. RNTI-E-3, 2: 697-702

Rothschild M.F., 2003. Approaches and challenges in measuring genetic diversity in pig. Arch. Zoot., 52: 129-135

Salako A.E., 2006. Principal component factor analysis of the morpho structure of immature uda sheep. Int. J. Morphol., 24 (4): 571-574

Toro M.A., Caballero A., 2005. Characterization and conservation of genetic diversity in subdivided populations. Phil. Trans. R. Soc. B Biol. Sci., 360: 1367-1378

Toro M.A., Meuwiessen T.H.E., Fernández J., Shaat I., Mäki-Tanila A., 2011. Assessing the genetic diversity in small farm animal populations. Animal, 5 (11): 1669-1683

Traore A., Tamboura H.H., Kabore A., Royo L.J., Fernandez I., Alvarez I., Sangare M., Bouchel D., Poivey J.P., Francois D., Toguyeni A., Sawadogo L., Goyache F., 2008. Multivariate characterization of morphological traits in Burkina Faso sheep. Small Rumin. Res., 80: 62-67

Wilson R.T., Durkin J.W., 1984. Age at permanent incisor eruption in indigenous goats and sheep in semi-arid Africa. Livest. Prod. Sci., 11: 451-455

Yakubu A., Ibrahim I.A., 2011. Multivariate analysis of morphostructural characteristics in Nigerian indigenous sheep. Ital. J. Anim. Sci., 10 83-86 


\section{Summary}

Laoun A., Harkat S., Benali R., Yabrir B., Hakem A., Ranebi D., Maftah A., Madani T., Da Silva A., Lafri M. Phenotypic characterization of the Rembi sheep of Algeria

The Algerian sheep stock remains little known, despite its high economic value and the very precious qualities of the local breeds which show exceptional adaptation under extreme environmental conditions. The phenotypic variability of Rembi, an endangered Algerian breed, was studied by multivariate discriminant analysis. Sampling took into account the diversity of production environments in the country according to FAO recommendations. In total 722 females and 60 males were phenotyped across a wide area including the cradle of the breed, using 21 quantitative and 12 qualitative variables. The results show strong phenotypic homogeneity, thus suggesting intrabreed homogeneity at the genetic level, to be confirmed by molecular analysis. Indeed, there was no evidence of a phenotypic structure when considering the farm status (private/State-owned), the climate zone (arid/semi-arid/subhumid), herd mobility (sedentary/semi-sedentary/transhumant) or the variety. Only the region factor discriminated subgroups within the breed. Trade, organized around major markets, promotes the flow of individuals between regions and seems responsible for the single phenotypic model. These results finely characterized the Rembi breed for the first time. Morphological indices place it in particular among meat breeds. This study is the first step toward the implementation of conservation programs for this breed, highly threatened by crosses with the Ouled-Djellal, the dominant Algerian breed.

Keywords: Rembi sheep, livestock breed, animal morphology, animal genetic resource, Algeria

\section{Resumen}

Laoun A., Harkat S., Benali R., Yabrir B., Hakem A., Ranebi D., Maftah A., Madani T., Da Silva A., Lafri M. Caracterización fenotípica de la raza ovina Rembi de Argelia

A pesar de su interés económico, el hato ovino argelino es mal conocido, aunque las razas locales se encuentran entre las más preciosas, mostrando adaptaciones excepcionales bajo condiciones ambientales extremas. La variabilidad fenotípica de la Rembi, raza argelina bajo riesgo, fue estudiada mediante un análisis discriminado multivariado. La muestra tomó en consideración la diversidad de los medio ambientes de producción presentes en el país, según las recomendaciones de la FAO. Se obtuvo el fenotipo de 722 hembras y 60 machos en total, sobre una zona vasta, incluyendo la cuna de la raza, mediante la utilización de 21 variables cuantitativas y 12 variables cualitativas. Los resultados muestran una fuerte homogeneidad fenotípica, sugiriendo la homogeneidad intra racial a nivel genético, a confirmar mediante análisis moleculares. En efecto, ninguna estructuración fenotípica fue demostrada, ya sea considerando el estado de la finca (privada/ estatal), la zona climática (árida/ semi árida/sub húmeda), la movilidad del hato (sedentario/semi sedentario/nómada) o incluso la variedad. Únicamente la consideración del factor región permitió demostrar sub grupos dentro de la raza. Los intercambios comerciales, organizados alrededor de los principales mercados, favorecen los flujos de individuos dentro de las diferentes regiones y parecen ser responsables de un modelo fenotípico único. Estos resultados permiten por la primera vez de caracterizar finamente la Rembi. Los índices morfológicos la sitúan particularmente entre las razas de carne. Este estudio es la primera etapa hacia la instauración de programas de conservación de esta raza tan amenazada por los cruces con Ouled-Djellal, raza argelina dominante.

Palabras clave: ovino Rembi, raza de ganado, anatomía animal, recurso genético animal, Argelia 